\title{
OPEN High-precision micro-displacement sensor based on tunnel magneto-resistance effect
}

\author{
Xuhu Wang ${ }^{1,2,3}$, Wang $\mathrm{Li}^{1,2,3}$, Li Jin ${ }^{1,2,3 凶}$, Meimei Gong ${ }^{2,3,4}$, Junqiang Wang ${ }^{1,2,3}$, \\ Yujie Zhong ${ }^{1,2,3}$, Yi Ruan ${ }^{1,2,3}$, Chunhong Guo ${ }^{1,2,3}$, Chenguang Xin ${ }^{1,2,3}$ \& Mengwei $\mathrm{Li}^{1,2,3 凶}$
}

\begin{abstract}
A high-precision micro-displacement sensor based on tunnel magneto-resistance effect is reported. We designed and simulated magnetic characteristics of the sensor, and employed chip-level Au-In bonding to implement low-temperature assembly of the TMR devices. We employed the subdivision interpolation technique to enhance the resolution by translating the sine-cosine outputs of a TMR sensor into an output that varies linearly with the displacement. Simultaneously, using the multibridge circuit method to suppress external magnetic and geomagnetic interference. Experimental result shows that the micro-displacement sensor has a resolution of $800 \mathrm{~nm}$, accuracy of $0.14 \%$ and a full-scale range of up to millimeter level. This work enables a high-performance displacement sensor, and provides a significant guide for the design of a micro-displacement sensor in practical applications.
\end{abstract}

Micro-displacement measurement with high resolution and accuracy has numerous potential applications, such as automotive ${ }^{1}$, robotic system ${ }^{2}$, positioning ${ }^{3}$ and medical applications ${ }^{4}$. Techniques based on capacitive ${ }^{5}$, optical $^{6-9}$, as well as magnetic sensors ${ }^{10-12}$ are widely used in non-contact displacement sensing. The capacitive type is susceptible to electromagnetic interference, and parasitic capacitance and fringe effects have been the major issues to improve the performance of the sensor ${ }^{13}$. Optical type is commonly used in displacement sensing due to its high accuracy and resolution, but it's expensive and is not suited in dusty or harsh environments. Since magnetic field is not sensitive to the presence of nonconductive contaminants (such as oil, dirt, dust, etc.), magnetic type has excellent performance in dirt immunity and durability in harsh environments except for lower power consumption and simple interfacing circuitries.

Based on the sensing mechanism, there are several schemes have been proposed for the magnetic displacement sensors. The magneto-inductive (MI) sensors measure the inductance of coils changed with the permeability of the core when the permanent magnet moves ${ }^{14}$. This kind of linear MI-type sensors with a range of tens of millimeters are available. The sensing element based on the Hall effect is used to measure the magnetic field of the permanent magnet, whose field has a predefined pattern as a function of the distance from the permanent magnet ${ }^{15-17}$. As a new alternative, the displacement can be measured by detecting the magnetic flux density, according to the linear relationship between the magnetic flux and the reciprocal of displacement ${ }^{18-23}$.

In this paper, a displacement sensor based on tunnel magneto-resistance (TMR) effect with sub-micrometer level resolution and millimeter level of operation range is demonstrated. We present the design, simulation, and fabrication of TMR-based displacement sensor. To avoid temperature failure of TMR devices during manufacturing, we employed chip-level Au-In bonding to implement low-temperature assembly of the TMR devices ${ }^{24-29}$. We innovatively exploit unique TMR devices arrangement to extract quadrature components, and interpolation circuit to conduct nonlinear A/D conversion, implementing high-resolution sensitivity of the displacement sensor. In addition, we also proposed to use a multi-bridge circuit to achieve suppression of the surrounding magnetic interference, thus enhancing the operation accuracy of the displacement sensor. The designed TMR displacement sensor provides high sensitivity while achieving a large operation range.

\footnotetext{
${ }^{1}$ School of Instrument and Electronics, North University of China, Taiyuan 030051, Shanxi, China. ${ }^{2}$ Academy for Advanced Interdisciplinary Research, North University of China, Taiyuan 030051, Shanxi, China. ${ }^{3}$ Nantong Institute of Intelligent Opto-Mechatronics, North University of China, Nantong 226000, Jiangsu, China. ${ }^{4}$ School of Information and Communication Engineering, North University of China, Taiyuan 030051, Shanxi, China. ${ }^{\square}$ email: jinli0307@163.com; Imwnuc@163.com
} 

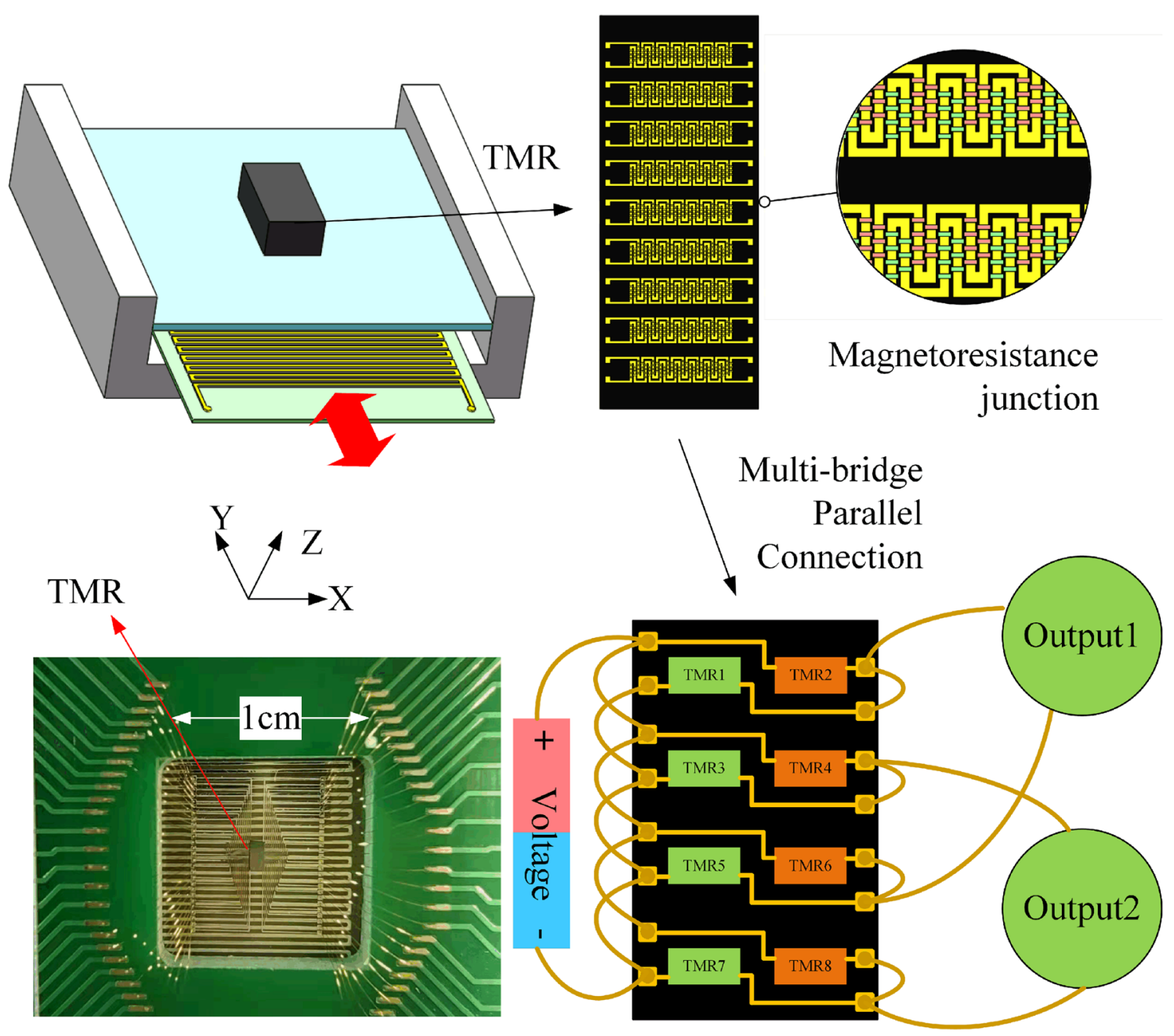

Figure 1. Schematic diagram of the TMR-based displacement sensor (Created by Microsoft Visio 2013 (15.0) and SOLIDWORKS(R) Premium 2016.).

\section{Principle of TMR displacement sensor}

A schematic diagram of the proposed displacement measurement system based on TMR effect is shown in Fig. 1. The TMR displacement sensor is composed of a copper electronic coil layer and TMR device layer. The electronic coil provides a uniform magnetic field in the $\mathrm{X}$-axis direction and highly gradient magnetic field in the Y-axis (sensing) direction. The magnetic resistance structure consists of several groups of TMR devices, which has two kinds of magnetic resistance junctions with opposite polarity. It's noteworthy that the magnetic field distribution and the sensitivity of the TMR devices directly decide the performance of TMR-based displacement sensor. During the operation of the proposed displacement sensor, it requires not only high gradient magnetic field in sensing direction, but also avoiding the electromagnetic interference. Thus, we employ a carefully designed electronic coil to provide the necessary magnetic field required to realize high sensitivity displacement measurements.

To further verify the feasibility of the design, a finite element method (FEM) simulation is implemented by COMSOL Multi-physics. In order to analyze the magnetic field characteristics generated by the electronic coils, we set the dimensional parameters with the optimal width of $200 \mu \mathrm{m}$, the thickness of $100 \mu \mathrm{m}$, and the distance of $200 \mu \mathrm{m}$ between the adjacent coils, as shown in Fig. $2 \mathrm{a}$. Figure $2 \mathrm{~b}$ shows the finite element simulation of the magnetic field distribution along the sense direction. According to the simulation results, We further extract the magnetic field amplitude along Y-axis and X-axis versus different height $H$ (ranging from 200 to $700 \mu \mathrm{m}$ with a step of $100 \mu \mathrm{m}$ ), shown in Fig. $2 \mathrm{c}$,d, respectively. From the simulation results, it can be seen that the magnetic field are basically symmetric and presents a quasi-sinusoidal distribution in the sense direction, with good consistency in the linear range, and the component of the magnetic field is almost a constant orthogonal to the sense direction. Moreover, the relationship between the magnetic field and the height $H$ shows that the closer to the electronic coils, the greater the magnetic field strength.

Due to the extremely high sensitivity of magnetic sensing of the TMR devices, it can successfully apply in this displacement measurement system. However, the electromagnetic interference will degrade the performance during its operation. Here we employ the multi-bridge circuit method to suppress external magnetic and geomagnetic interference and avoid the integration error of the TMR devices effectively, as shown in Fig. 1. The differential voltage output of any two adjacent bridges in the multi-bridge circuits is ${ }^{17}$ : 
(a)
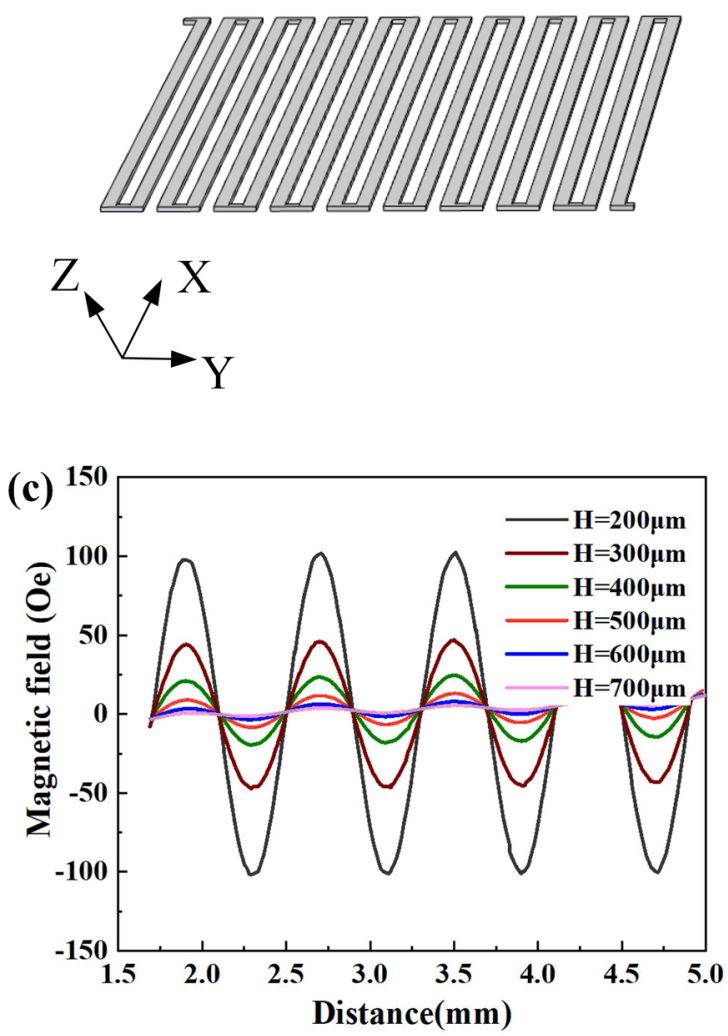

(b)
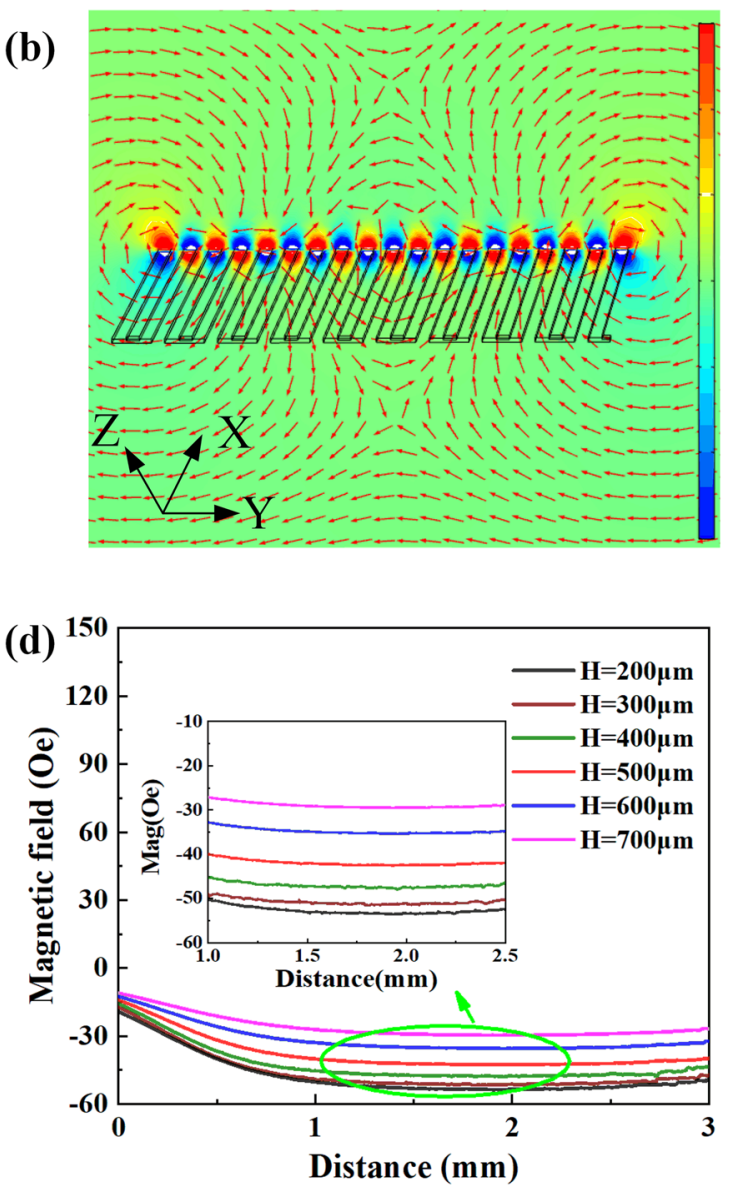

Figure 2. Magnetic field distribution analysis. $H$ is the distance between electronic coil layer and TMR devices layer, ranging from 200 to $700 \mu \mathrm{m}$ with a step of $100 \mu \mathrm{m}$. (a) Model of the electronic coil (Created by Microsoft Visio 2013 (15.0) and SOLIDWORKS(R) Premium 2016.). (b) The magnetic field distribution along the sense direction. (c) The magnetic field along the sense direction (Y-axis) versus different layer distance $H$. (d) The magnetic field along the $\mathrm{X}$-axis versus different layer height $H$.

$$
V=-\frac{K A V_{0}}{R_{0}} \sin \frac{\pi(2 y+d)}{2 D} \sin \frac{\pi d}{2 D}
$$

where $V_{0}$ is the static voltage and $R_{0}$ is the static resistance of the TMR, $K$ is the sensitivity of the TMR devices, $A$ is the amplitude of magnetic feld, $D$ is the line spacing of electric coils, $d$ is the spacing distance of the adjacent bridge circuits and $y$ is the displacement along the sense direction. From Eq. (1), two sinusoidal signals with a phase difference of $90^{\circ}$ can be obtained by the movement of the electromagnetic coils which will cause magnetic field to change periodically. To further improve the sensitivity and the range of the displacement sensor, it's necessary to apply relevant interpolation circuits.

\section{Fabrication processing}

To implement the displacement sensor assembly, it's necessary to connect the TMR devices to the supporting substrate via metal bonding. However, there exists a compromise between the shear strength and the bonding temperature due to the temperature failure of TMR devices. Due to the low-melting point of In, wafer-level Au-In bonding can be performed at considerably lower temperature than other metal eutectic bondings.

The fabrication process of the bonding structure is shown in Fig. 3a-j. A 100-nm-thick $\mathrm{SiO}_{2}$ passivation layer was deposited on silicon wafer (Fig. 3a) surface by plasma-enhanced chemical vapor deposition (PECVD), as shown in Fig. 3b. A 10-nm-thick Cr adhesive layer and 200-nm-thick Au are deposited on the $\mathrm{SiO}_{2}$ layer through magnetron sputtering. The multilayered-metal $(\mathrm{Cr} / \mathrm{Au})$ has been employed to form the electrode and bonding pads by lift-off technique (Fig. 3c). A $1.5 \mu \mathrm{m}$ thickness of In layer were evaporated onto the bonding pads (Fig. 3d) and then $4 \mu \mathrm{m}$ thickness of photoresist spin-coated on the bonding pads (Fig. 3e). With the protection of photoresist, we scribe the silicon wafer into pieces and reduce the thickness to $50 \mu \mathrm{m}$ (Fig. $3 \mathrm{f}-\mathrm{g}$ ). Finally, the alignment of the bonding die and the TMR devices (Fig. 3h) was performed to achieve the wafer-level Au-In bonding (Fig. 3j) after the removal of photoresist (Fig. 3i). The corresponding fabricated wafer is shown in Fig. 4a. A micrograph of the bonding pads and the image of wafer-level Au-In bonding with TMR are also shown in Fig. 4b,c, respectively. 


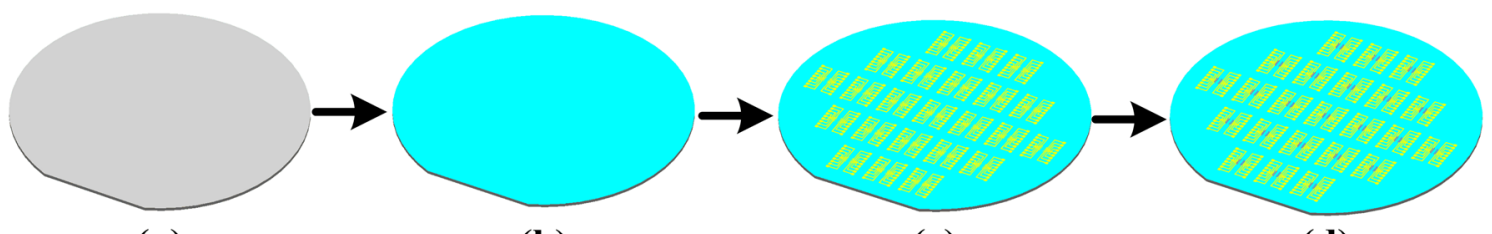

(a)

(b)

(c)

(d)

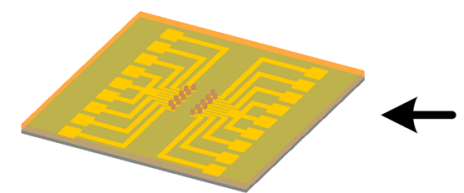

(g)

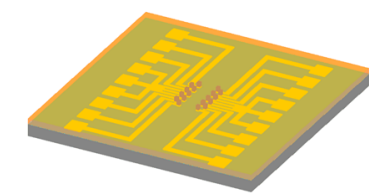

(f)

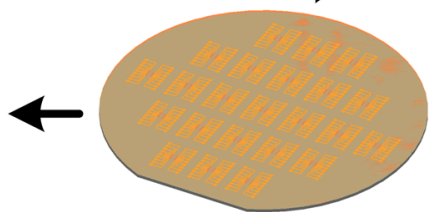

(e)

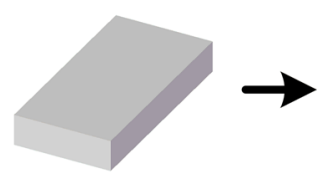

(h)
4

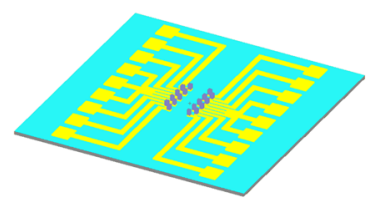

(i)
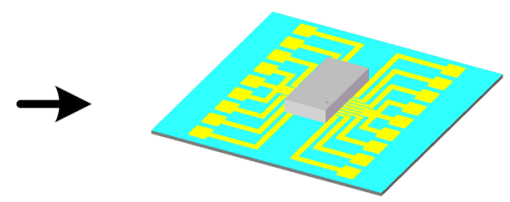

(j)

Figure 3. Fabrication processing flow of the bonding structure. (a) Sample wafer. (b) $\mathrm{SiO}_{2}$ passivation layer was deposited on the surface by PECVD. (c) Sputtering of the electrode and bonding pads. (d) In layer evaporated onto the bonding pads. (e) Photoresist coated. (f) Scribing. (g) Reducing the thickness. (h) TMR device. (i) Photoresist removed. (j) Wafer-level Au-In bonding. (Created by Microsoft Visio 2013 (15.0) and SOLIDWORKS(R) Premium 2016.).

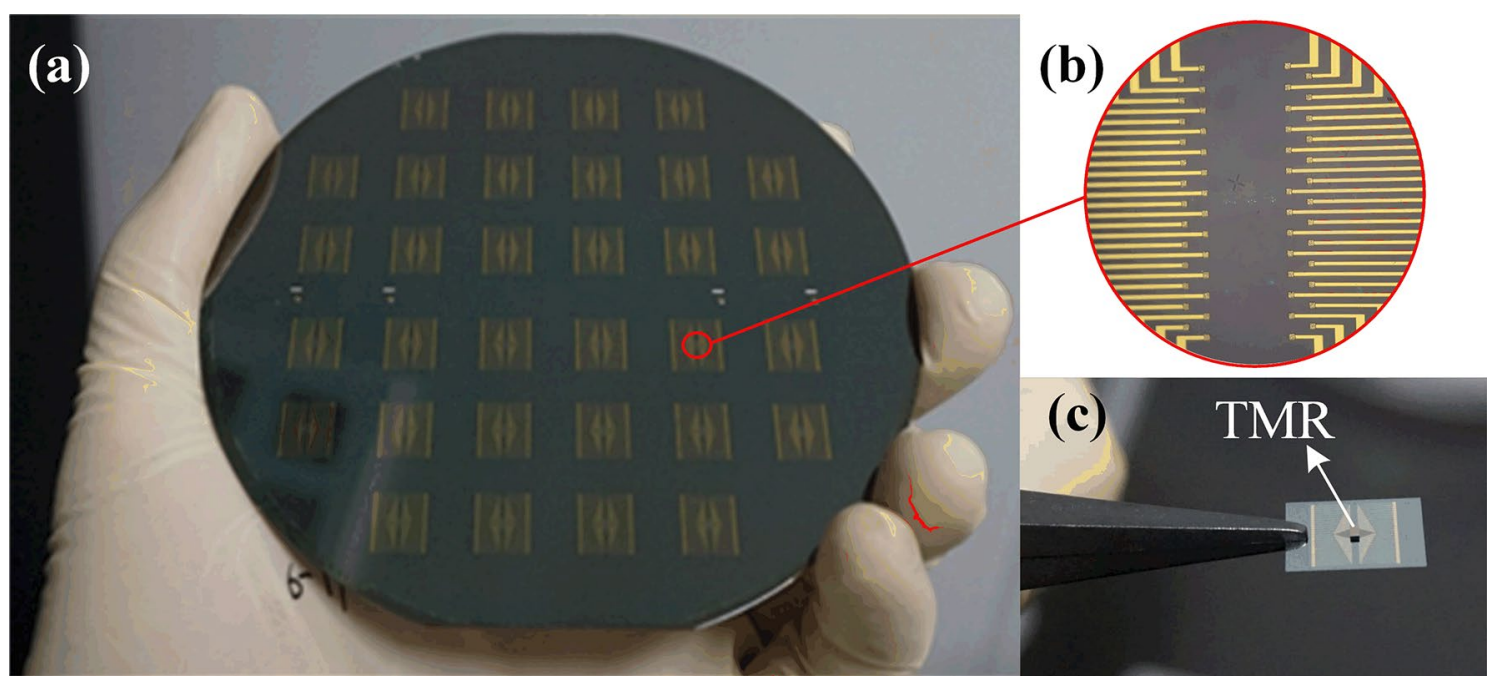

Figure 4. (a) Photograph of the fabricated 4-inch silicon wafer. (b) Micrograph of the bonding pads (Created by Microsoft Visio 2013 (15.0)). (c) Image of wafer-level Au-In bonding with TMR.

To ensure there is pure In at the surface prior to bonding, the $\mathrm{AuIn}$ growth should be limited by $\mathrm{Au}$, and the required In/Au thickness ratio should be larger than the ratio corresponding to a complete conversion of all $\mathrm{Au}$ and In into $\mathrm{AuIn}_{2}$, estimated to 3.1 by:

$$
\frac{y(\operatorname{In})}{y_{(}(A u)}=\frac{m(\operatorname{In}) / \rho(\operatorname{In})}{m(A u) / \rho(A u)}=\frac{0.54 / 7.31}{0.46 / 19.3}=3.1
$$

where $m_{I_{n}}$ and $m_{A_{u}}$ are the mass of In and Au that are consumed into AuIn $2,0.54$ is the weight ratio of In in $\mathrm{AuIn}_{2}, 0.46$ is the weight ratio of $\mathrm{Au}$ in $\mathrm{AuIn}_{2}, \rho_{I_{n}}$ and $\rho_{I_{n}}$ are the densities of In and $\mathrm{Au}$, respectively. Therefore, we deposited $1 \mu \mathrm{m}, 1.5 \mu \mathrm{m}, 2 \mu \mathrm{m}, 2.5 \mu \mathrm{m}$ In onto $0.2 \mu \mathrm{m}$ Au giving a In/Au ratio significantly larger than the requirement of 3.1 .

Due to the TMR device is vulnerable to temperature, we analyze the bonding temperature that will affect the shear strength of Au-In bonding, as shown in Fig. 5. The schematic configuration of the shear test is shown in 
(a)

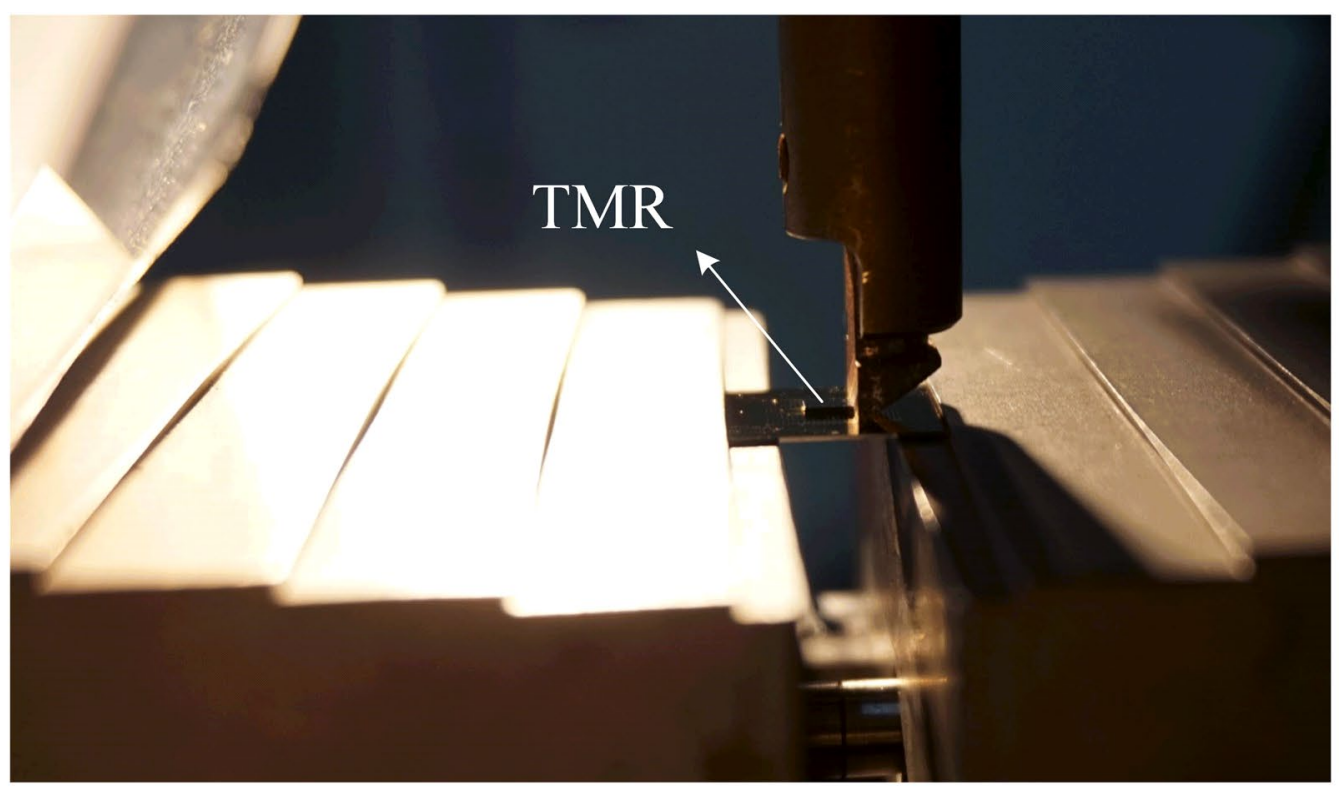

(b)

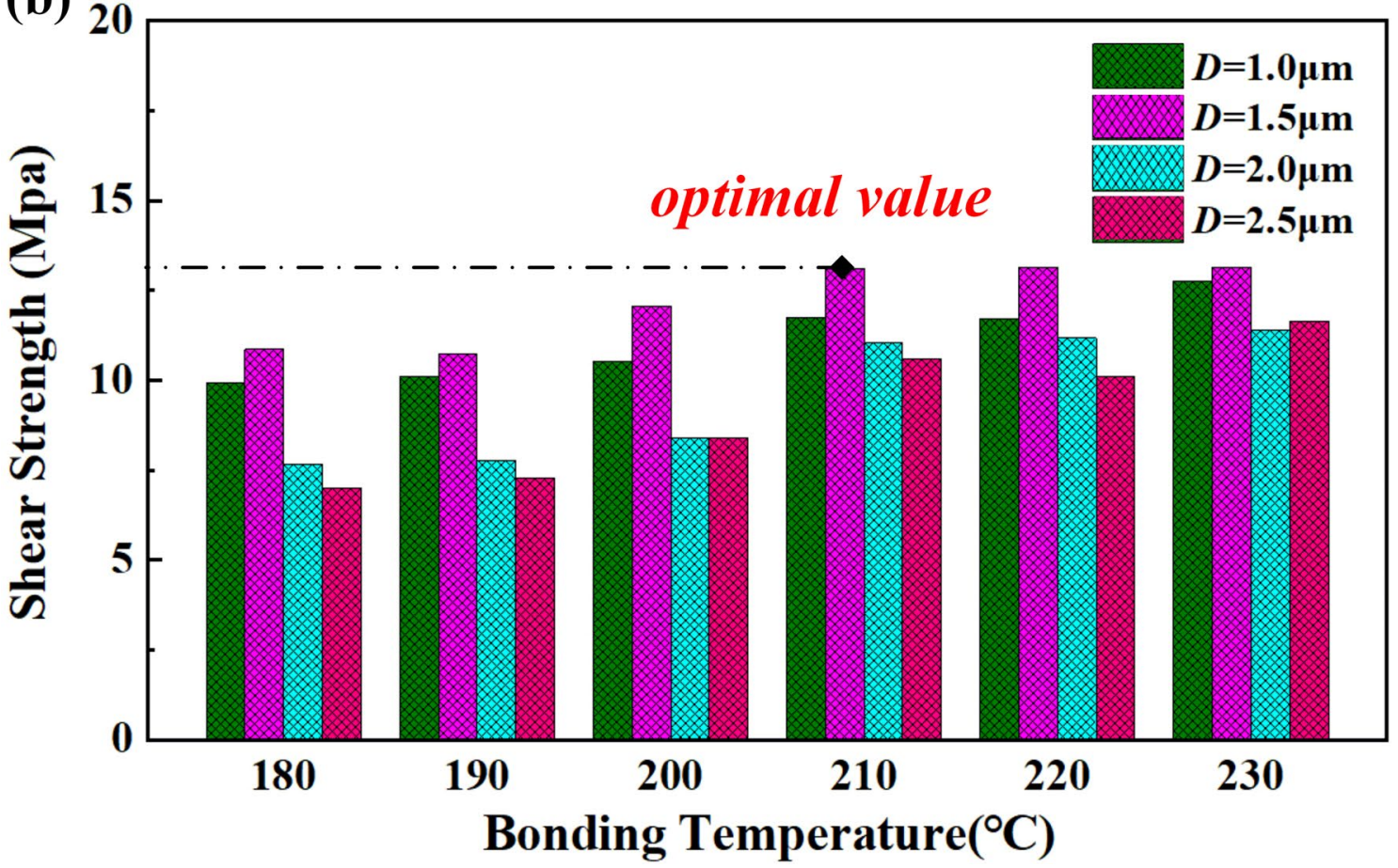

Figure 5. Shear test results. (a) Illustration of shear test configuration. (b) The shear strength as function of bonding temperature with different In thickness $D$.

Fig. 5a. At a shear test pressure of $20 \mathrm{MPa}$ and bonding time of $5 \mathrm{~min}$, it's obvious that the increment in shear strength is significant while the bonding temperature changes from 453 to $503 \mathrm{~K}\left(180-230^{\circ} \mathrm{C}\right)$. There exists an optimal In thickness, as shown in Fig. 5b, the shear strength of the bonding layer is up to more than $10 \mathrm{MPa}$ with the thickness of $1.5 \mu \mathrm{m}$. According to the remanence temperature coefficient, the magnetism of the magnetic material in the TMR decreases with increasing temperature which directly affects the TMR performance. It will mostly recover after cooling when the temperature rises within certain temperature range. It is experimentally demonstrated that the TMR will cause irreversible process when the temperature increases further more than $493 \mathrm{~K}\left(220^{\circ} \mathrm{C}\right)$ and will damage as higher temperature, especially larger than $523 \mathrm{~K}\left(250^{\circ} \mathrm{C}\right)$. According to the above analysis of shear strength dependence, $\mathrm{Au}$-In bonding experiment is performed at a lower temperature $483 \mathrm{~K}\left(210^{\circ} \mathrm{C}\right)$ with the thickness of $1.5 \mu \mathrm{m}$.

Figure 6 shows the SEM/EDS image of fracture surface of bonded sample. We observe three different positions in fracture surface, as shown in Fig. 6a-c. The positions of this fracture correspond to the bond interface between the substrate and the compound layer, indicating that rupture occurs at adhesive layer rather than cohesive fracture, compared with the other fracture surface shown in Fig. 6d. From the Fig. 6a,c, two Au-In intermetallic 

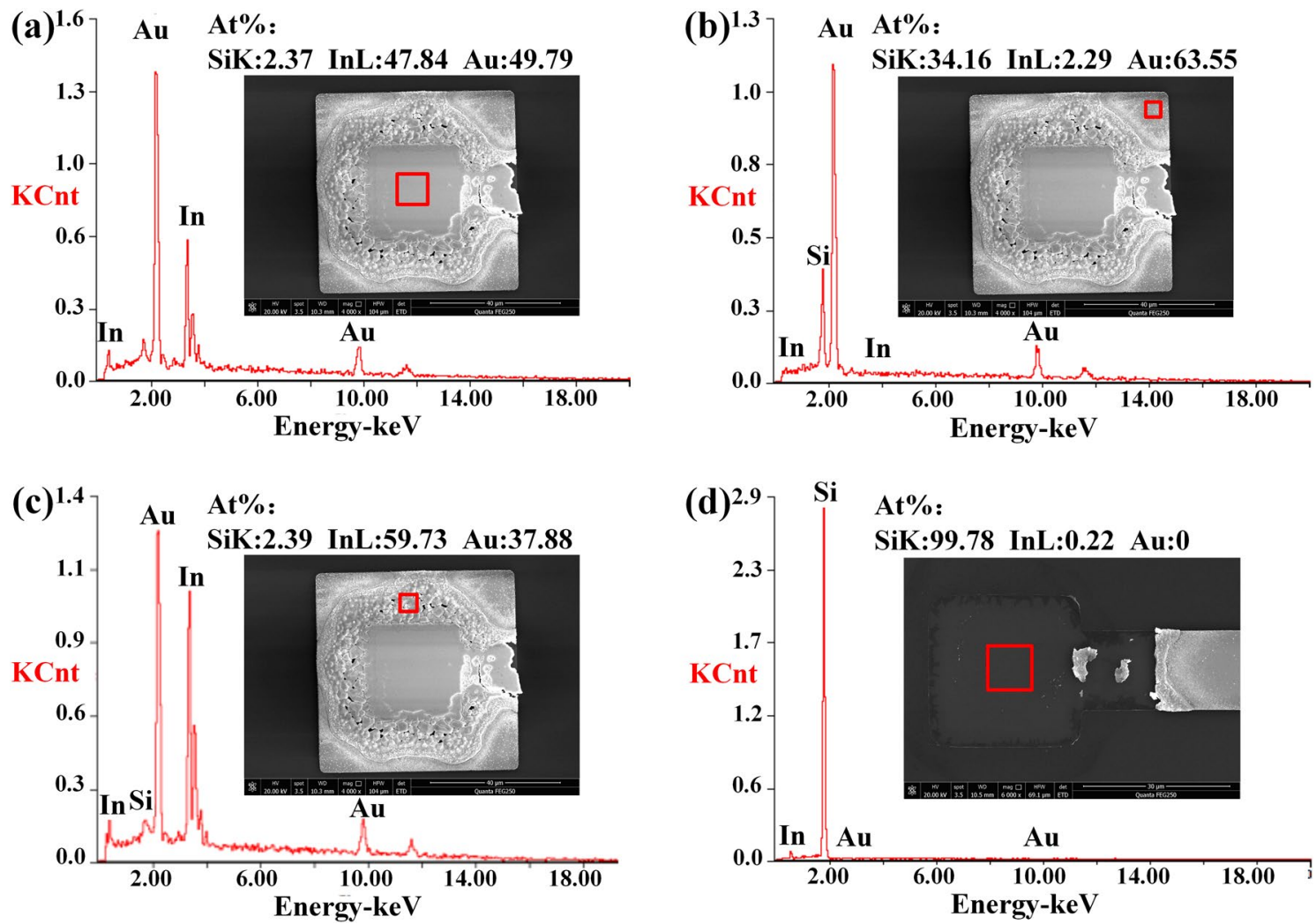

Figure 6. SEM/EDS image of fracture surface of bonded sample. Three different fracture positions were observed in $(\mathbf{a}-\mathbf{c})$, compared with the other fracture surface shown in $(\mathbf{d})$, the fracture occurs at adhesive layer.
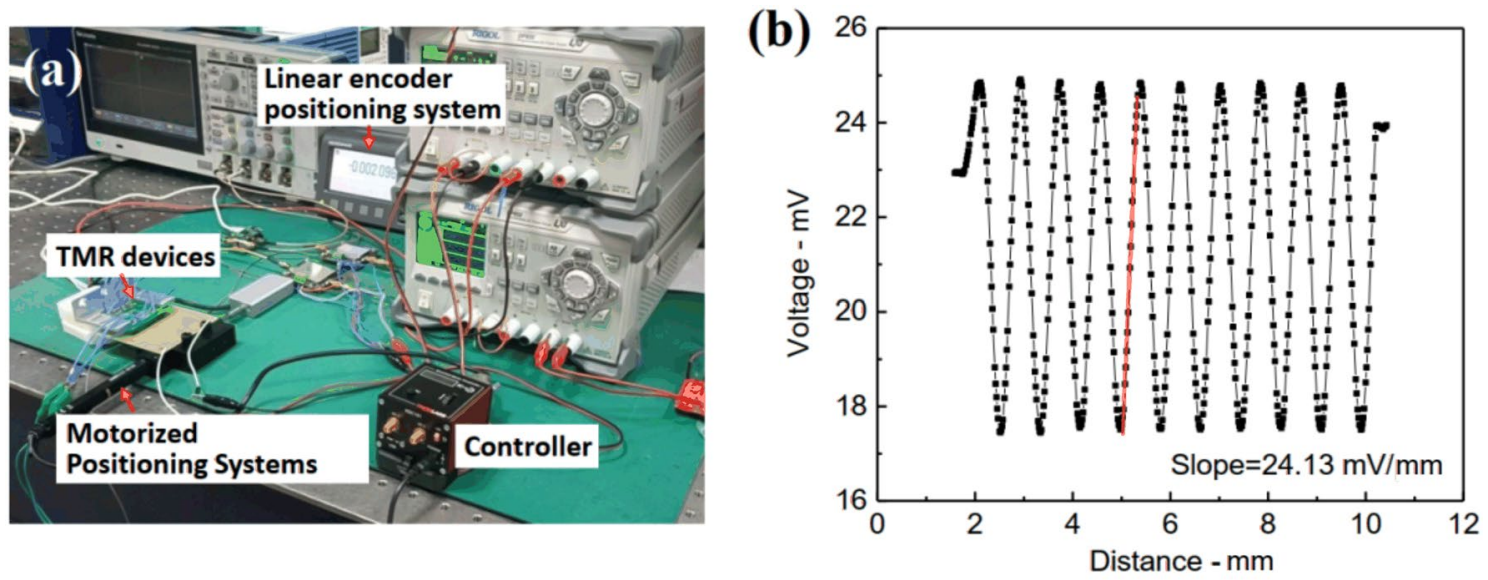

Figure 7. Experimental tests of the TMR devices. (a) Experimental setup. (b) The magnetic-resistance sensitivity of the TMR devices.

compounds (AuIn and $\mathrm{AuIn}_{2}$ ) are present with In mole fraction 50 and $30 \mathrm{pct}$, demonstrating produced stable bonds in TMR fabrication process.

\section{Results and discussion}

The displacement sensor behavior is not only dependent on the generated field but the sensitivity of TMR devices. It shows a linear relationship between the magneto-resistance and the external field, therefore, the influence of hysteresis phenomenon is negligible. To represent the characteristic of the TMR devices, we measure the magnetic-resistance sensitivity of the TMR devices with the operation voltage of $5 \mathrm{~V}$, as shown in Fig. 7 . The electric coil is fixed on the motorized positioning system, we record the output of the TMR device when moving the positioning system, as shown in Fig. 7a. According to the gradient of the magnetic field of $0.763 \mathrm{Oe} / \mathrm{mm}$, the magnetic-resistance sensitivity of the TMR devices is $31.62 \mathrm{mV} / \mathrm{Oe}$ from the linear fitting of Fig. $7 \mathrm{~b}$. 


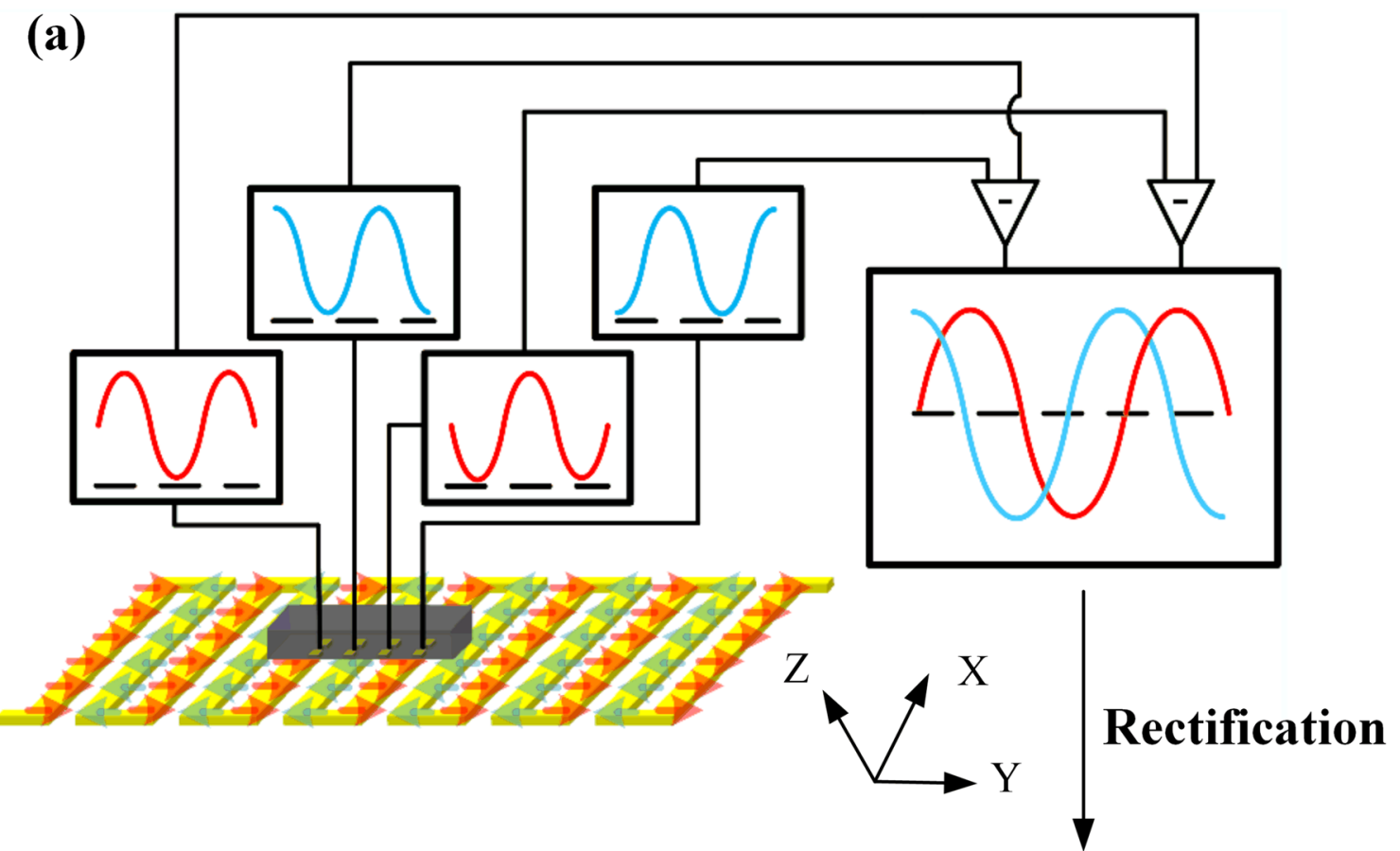

(c)

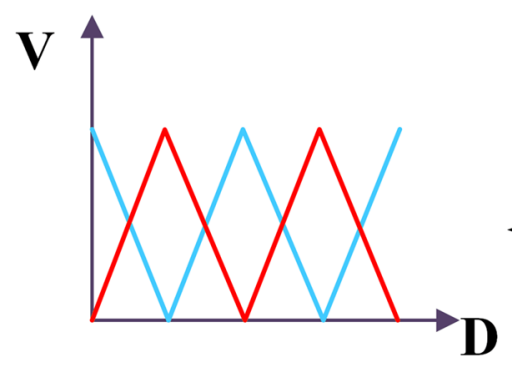

(d)

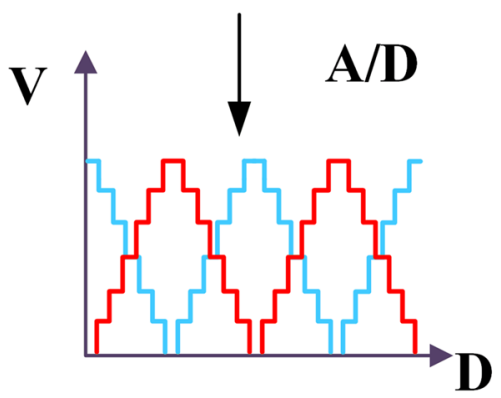

(b)
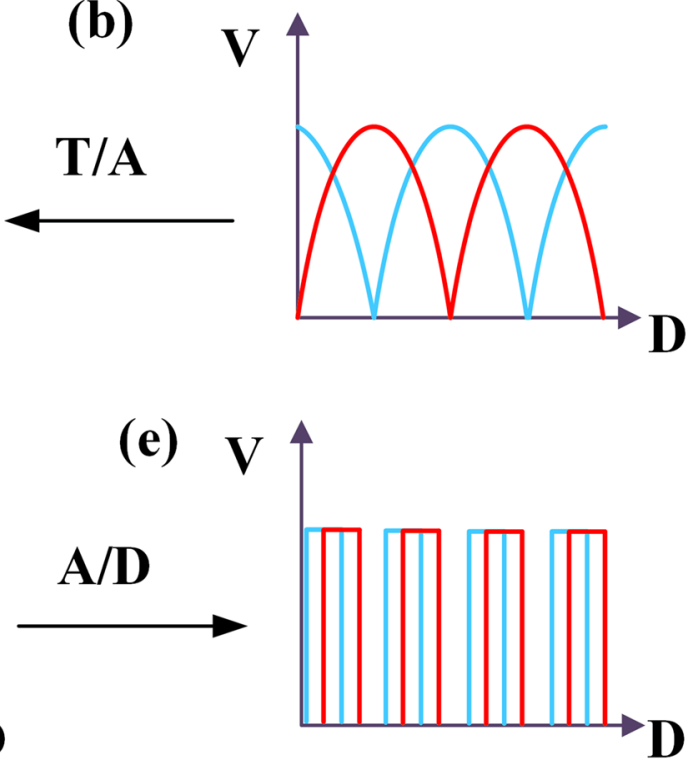

Figure 8. Principle of subdivision interpolation circuit. (a) A couple of sinusoids with phase difference of $90^{\circ}$ (Created by Microsoft Visio 2013 (15.0) and SOLIDWORKS(R) Premium 2016.). (b-d) Process of the interpolation circuits converting the two sinusoidal signals into an industry standard incremental quadrature digital signal.

Due to the distribution of the magnetic field is a quasi-sine function curve in the sense direction, we arrange TMRs of the bridge circuits equidistant and the spacing distance is designed to a quarter of the period of the sinusoidal magnetic field. When the coils layer moves along the sense direction, two sinusoidal signals with a phase difference of $90^{\circ}$ can be obtained from the output port. To enhance higher resolution of the displacement sensor, the interpolation circuit is employed to process the two sinusoidal signals to an industry standard incremental quadrature digital signal, as shown in Fig. 8. Here, we employed IC-TW8 chip from IC-Haus to interpolate the output signals. According to the dimensional parameters of electric coils, the output signal has a period of $800 \mu \mathrm{m}$, the resolution of displacement sensor can reach to $800 \mathrm{~nm}$ after 1000 times subdivision $^{30,31}$.

Figure 9a shows the input signal of the interpolation circuit with a digital oscilloscope, the red and black curves are quadrature signals with a phase difference of $90^{\circ}$. The red and black square wave signals are the relevant output of the interpolation circuit, which can accurately output the incremental AB quadrature digital signal, as shown in Fig. 9b. By comparing different interpolation factors, the final interpolation factor $N$ is chosen to be 1000. Due to the factors (such as the temperature drift of the operational amplifier, DC offset error and gain error) will directly affect the accuracy of interpolation circuit, we employ the linear encoder positioning system 

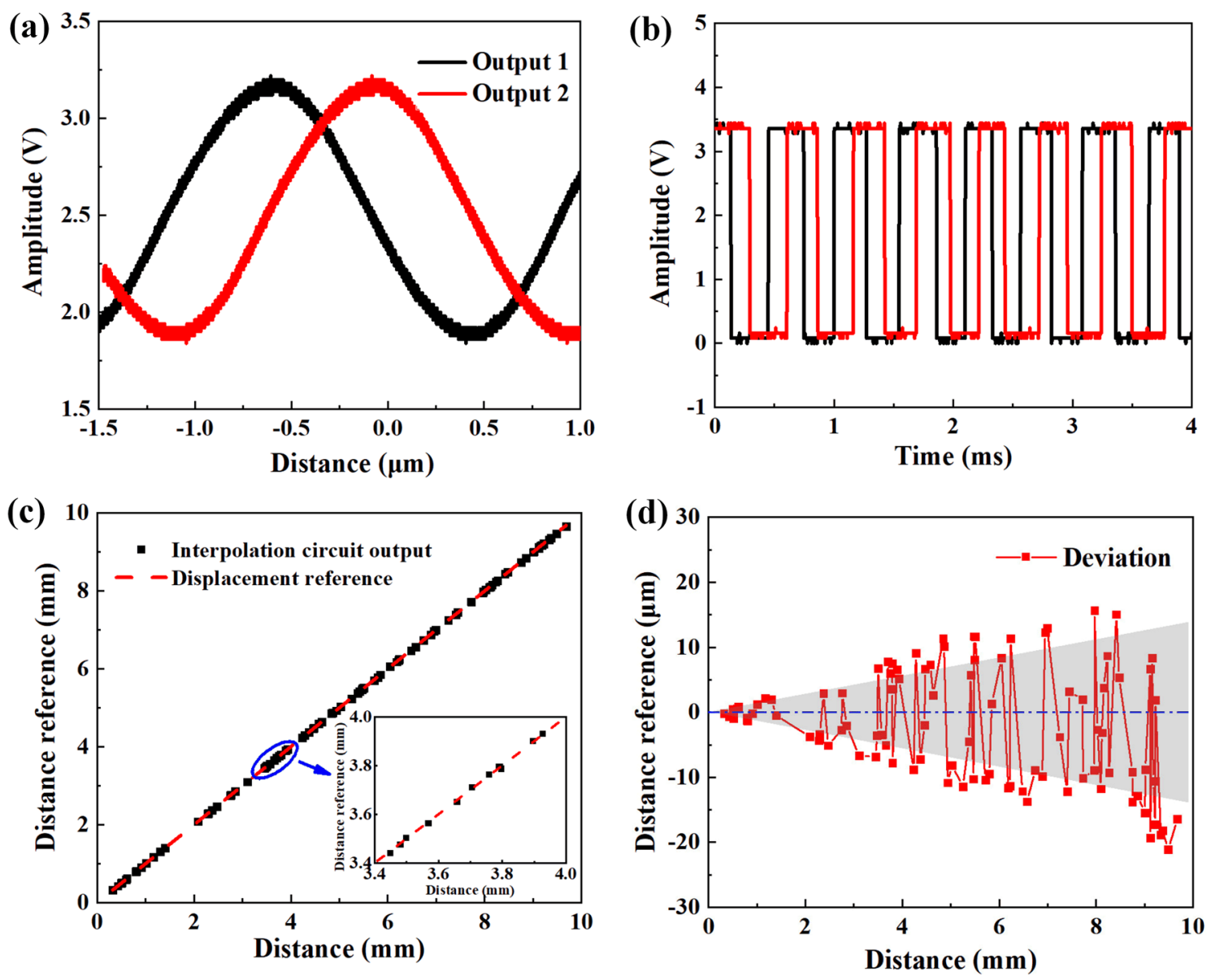

Figure 9. (a) Two sinusoidal signals with quadrature phase shift. (b) Standard incremental AB quadrature digital signal. (c) Comparison of the linear encoder positioning system and displacement output of the interpolation circuit. (d) Error distribution curve.

produced by Heidenhain (Heidenhain MT1281, with the resolution of $0.5 \mathrm{~nm}$ and accuracy of $\pm 0.2 \mu \mathrm{m}$ ) to move along the sliding guide and use it as a displacement reference. Figure $9 \mathrm{c}$ shows the comparison the output of linear encoder positioning system and the interpolation circuit. It shows that the result of the displacement measurement agrees well with the reference data. From the error distribution curve, the average error values are $0.14 \%$ by comparing the linear encoder positioning system and the displacement of the interpolation circuit output, as shown in shaded area of Fig. $9 \mathrm{~d}$.

Due to the maximum interpolation factor of the interpolation circuit is 10,000 , it's not the highest level achievable in our research group currently, the resolution can reach to nanometer level if possible in the near future. Meanwhile decreasing the period of the current coil and reducing the height of TMR devices can effectively improve the measurement resolution.

\section{Conclusion}

We proposed a novel high-sensitivity micro-displacement sensor based on tunnel magneto-resistance effect. The simulations are implemented to determine the magnetic characteristics of the device and exhibit the magneticresistance sensitivity of $31.62 \mathrm{mV} / \mathrm{Oe}$. We employed the $1000 \times$ interpolation circuit and multi-bridge tunneling magneto-resistance detection to achieve the TMR-based displacement sensor with sub-micrometer level resolution and millimeter level of operation range. With the deepening of research, the sensitivity of the displacement sensor can be further enhanced by reducing the distance between electronic coil layer and TMR devices layer, increasing the interpolation factor and decreasing the duty cycle of the electric coils. This kind of TMR-based displacement sensor possesses the advantages of high resolution, low energy consumption and is suitable for various applications in the future.

\section{Data availability}

All data generated or analysed during this study are included in this published article. 


\section{References}

1. Sandra, K. R., Georg, B. \& Kumar, J. V. Combined variable reluctance-hall effect displacement sensor. IEEE Trans. Instrum Meas. 67(5), 1169-1177 (2017).

2. Jeon, H. et al. Automatic classification of tremor severity in parkinson's disease using a wearable device. Sensors. 17(9), 2067 (2017).

3. Liu, X. K., Peng, K., Chen, Z. R., Pu, H. J. \& Yu, Z. C. A new capacitive displacement sensor with nanometer accuracy and long range. IEEE Sens J. 16(8), 2306-2316 (2016).

4. Zhao, H. B. et al. High-precision microdisplacement sensor based on zeroth-order diffraction using a single-layer optical grating. Appl Opt. 59(1), 16-21 (2020).

5. Pu, H. J., Liu, H. Z., Liu, X. K., Peng, K. \& Yu, Z. C. A novel capacitive absolute positioning sensor based on time grating with nanometer resolution. Mech. Syst. Signal Process. 104, 705-715 (2018).

6. Li, M. W. et al. Large-scale range diffraction grating displacement sensor based on polarization phase-shifting. Appl. Opt. 59(2), 469-473 (2020).

7. Hu, P. C., Chang, D., Tan, J. B., Yang, R. T. \& Fu, H. J. Displacement measuring grating interferometer: a review. Front. Inf. Technol. Electron. Eng. 20(5), 631-654 (2019).

8. Lin, J., Guan, J., Wen, F. \& Tan, J. B. High-resolution and wide range displacement measurement based on planar grating. Comp. Opt. Commun. 404, 132-138 (2017).

9. Ye, G. Y. et al. Optimal design of a reflective diffraction grating scale with sine-trapezoidal groove for interferential optical encoders. Opt. Lasers Eng. 134, (2020).

10. Zhang, J. K., Wang, R. B., Deng, Z. Y. \& Kang, Y. H. A displacement sensing method based on alternating current magnetic flux measurement. Meas. Sci. Technol. 29(8), (2018).

11. Sun, J. J. \& Zhang, Y. A novel integrated structure with a radial displacement sensor and a permanent magnet biased radial magnetic bearing. Comp. Sensors. 14(2), 1950-1960 (2014).

12. Peng, K. Y. \& Chang, J. K. Effects of assembly errors on axial positioning accuracy for rotating machinery with magnetoresistancebased magnetic encoders. Microsyst. Technol. 27(6), 2507-2514 (2021).

13. George, B., Tan, Z. C. \& Nihtianov, S. Nihtianov, advances in capacitive, eddy current, and magnetic displacement sensors and corresponding interfaces. Comp. IEEE Trans. Ind Electron. 64(12), 9595-9607 (2017).

14. Wu, Z. et al. A magnetoelectric laminate based passive micro-displacement sensor. Phys. Status Solidi Rapid Res. Lett. 6(12), 469-471 (2012).

15. Li, C., Yang, B., Guo, X. \& Chen, X. R. Design, analysis and simulation of a mems-based gyroscope with differential tunneling magnetoresistance sensing structure. Sensors. 20(17), 4919 (2020)

16. Li, M. W., Ding, X. C. \& Qin, S. Y. Design of a novel lower-noise tunneling magnetoresistance micromachined gyroscope. Microsyst. Technol. 25(4), 1447-1454 (2019).

17. Jin, L., Qin, S. Y., Zhang, R. \& Li, M. W. High-sensitivity tunneling magneto-resistive micro-gyroscope with immunity to external magnetic interference. Sci. Rep. 10(1), 1-10 (2020).

18. Polovy, H. et al. Noise of mgo-based magnetic tunnel junctions. J. Magn. Magn. Mater. 322(9-12), 1624-1627 (2010).

19. Lee, C. C., Yen, Y. S. \& Lai, C. H. Alignment-free sensing module for absolute and incremental lines in linear positioning system based on tunneling-magnetoresistance sensors. Sensors. 21(12), 4137 (2021).

20. Hu, J. F., Pan, M. H., Tian, W. G., Chen, D. X. \& Luo, F. L. Integrating magnetoresistive sensors with microelectromechanical systems for noise reduction. Appl. Phys. Lett. 101(23), (2012).

21. Deak, J. G., Zhou, Z. M. \& Shen, W. F. Tunneling magnetoresistance sensor with pt level 1/f magnetic noise. AIP Adv. 7(5), 056676 (2017).

22. $\mathrm{Mu}, \mathrm{X}$. H. et al. A new rapid detection method for ricin based on tunneling magnetoresistance biosensor. Sens Actuators B Chem. 284, 638-649 (2019).

23. Wang, C. et al. A novel emt system based on tmr sensors for reconstruction of permeability distribution. Meas. Sci. Technol. 29(10), (2018).

24. Luu, T. T., Hoivik, N., Wang, K. Y., Aasmundtveit, K. E. \& Vardøy, A. S. B. Characterization of wafer-level au-in-bonded samples at elevated temperatures. Metall. Mater. Trans. A. 46(6), 2637-2645 (2015).

25. Liu, M. M. et al. Research on preparation and properties of isothermal solidification cu-sn high temperature solder paste. JIAET 2018, 1-5 (2018)

26. Shieu, F. S., Chen, C. F., Sheen, J. G. \& Chang, Z. C. Intermetallic phase formation and shear strength of a au-in microjoint. Thin Solid Films. 346(1-2), 125-129 (1999).

27. Tollefsen, T. A., Løvvik, M. O., Aasmundtveit, K. \& Andreas, L. Effect of temperature on the die shear strength of a au-sn slid bond. Metall. Mater. Trans. A Phys. Metall. Mater. Sci. 44(7), 2914-2916 (2013).

28. Wang, Y. Q. et al. Micro-fabricated alkali vapor cells sealed at low temperature using asymmetric au-in transient liquid phase (tlp) bonding. Jpn. J. Appl. Phys. 58(SD), 03 (2019).

29. Hao, F. F., Wang, J. Q. \& Li, M. W. Low-temperature anodic bonding for wafer-level al-al interconnection in mems grating gyroscope. IEEE Trans. Compon. Packaging. Manuf. Technol. 11(1), 19-24 (2020).

30. Lin, J., Guan, J., Wen, F. \& Tan, J. B. High-resolution and wide range displacement measurement based on planar grating. Opt. Commun. 404, 132-138 (2017).

31. Cheng, F. \& Fan, K. C. Linear diffraction grating interferometer with high alignment tolerance and high accuracy. Appl. Opt. 50(22), 4550-4556 (2011).

\section{Author contributions}

Conceptualization, X.W. and L.J.; methodology, X.W., J.W.and L.J.; software, X.W.; validation, X.W., W.L., Y.Z., Y.R. and M.G.; formal analysis, X.W.; investigation, W.L.and C.G.; resources, M.L.; data curation, X.W.; writingoriginal draft preparation, X.W.; writing-review and editing, X.W., L.J. and C.X.; visualization, L.J.; supervision, C.X.; project administration, M.L.; funding acquisition, X.W. All authors have read and agreed to the published version of the manuscript.

\section{Funding}

National Natural Science Foundation of China (Grant No. 62005253); Shanxi Province Science Foundation for Youths (201901D211277); Scientific and Technological Innovation Programs of Higher Education Institutions in Shanxi (2020L0267); Science Foundation of North University of China (XJJ201901).

\section{Competing interests}

The authors declare no competing interests. 


\section{Additional information}

Correspondence and requests for materials should be addressed to L.J. or M.L.

Reprints and permissions information is available at www.nature.com/reprints.

Publisher's note Springer Nature remains neutral with regard to jurisdictional claims in published maps and institutional affiliations.

(c) (1) Open Access This article is licensed under a Creative Commons Attribution 4.0 International License, which permits use, sharing, adaptation, distribution and reproduction in any medium or format, as long as you give appropriate credit to the original author(s) and the source, provide a link to the Creative Commons licence, and indicate if changes were made. The images or other third party material in this article are included in the article's Creative Commons licence, unless indicated otherwise in a credit line to the material. If material is not included in the article's Creative Commons licence and your intended use is not permitted by statutory regulation or exceeds the permitted use, you will need to obtain permission directly from the copyright holder. To view a copy of this licence, visit http://creativecommons.org/licenses/by/4.0/.

(C) The Author(s) 2022 